\title{
Population synthesis of Galactic subdwarf B stars
}

\author{
G. Nelemans
}

Received: 5 November 2009 / Accepted: 8 April 2010 / Published online: 9 June 2010

(C) The Author(s) 2010. This article is published with open access at Springerlink.com

\begin{abstract}
I briefly review the method of population synthesis of binary stars and discuss the preliminary results of a study of the Galactic population of subdwarf B stars. In particular I focus on the formation of (apparently) single sdB stars and their relation to (apparently) single helium-core white dwarfs. I discuss the merits of mergers of two helium white dwarfs and interactions with sub-stellar companions for explaining these single objects. A preliminary conclusion is that the current observations suggest both mechanisms may contribute, but that the helium white dwarfs are likely formed in majority from interactions with sub-stellar companions.
\end{abstract}

Keywords Binary stars · Subdwarf B stars · Galactic population

\section{Introduction}

In a volume like this one, no lengthy introduction to subdwarf B ( $\mathrm{sdB}$ ) stars is needed, as many aspects of their nature are discussed in the different contributions. Instead I will briefly describe the motivation to study the Galactic population of sdB stars. Because rather special circumstances are needed to form an sdB star (the hydrogen envelope needs to be expelled at the right moment), they offer one of the best populations to constrain binary-evolution models, in particular the common-envelope phase (e.g. Han et al. 2003; Hu et al. 2007).

G. Nelemans $(\bowtie)$

Department of Astrophysics, Radboud University Nijmegen, P.O. Box 9010, 6500 GL, Nijmegen, The Netherlands

e-mail: nelemans@astro.ru.nl
Secondly, the fact that some sdB stars are pulsators allows asteroseismological determination of accurate masses and internal structure, which provides tests of stellar evolution and in particular an excellent way to constrain their formation (e.g. Fontaine et al. 2008, and contributions of Van Grootel and $\mathrm{Hu}$ in this volume).

Finally, the relative brightness of $\mathrm{sdB}$ stars allows us to study them to much larger distances than the other objects that probe the late phases of evolution of low- and intermediate-mass stars, the white dwarfs. Indeed, they are likely responsible for much of the UV light observed in elliptical galaxies (e.g. Han et al. 2007, and Han, this volume).

However, I think the close link of sdB stars with white dwarfs-either low-mass helium-core white dwarfs as "failed" sdB stars, or the low-mass $\mathrm{C} / \mathrm{O}$ core white dwarf descendants of sdB stars-deserves more attention. In the remainder of this paper I will discuss the principle of population synthesis (Sect. 2), a model for the Galactic population of sdB stars (Sect. 3) a discussion of sdB stars in relation to white dwarfs in Sect. 4 and the probability of finding sdB stars in binaries with neutron stars or black holes (Sect. 5).

\section{Population synthesis}

One of the ways to constrain the binary evolution using sdB stars, is using so called population synthesis (for a general description of the different population synthesis codes see Kornilov and Lipunov 1983; Dewey and Cordes 1987; Lipunov and Postnov 1988; de Kool 1990; Yungelson and Tutukov 1991; Kolb 1993; Pols and Marinus 1994; Portegies Zwart and Verbunt 1996; Vanbeveren et al. 1997; Fryer et al. 1998; Nelemans et al. 2001; Hurley et al. 2002; Han et al. 2003; Belczynski et al. 2008). The basic concept 
of this method is that a large number of binary evolution scenarios are calculated using (approximations to) the evolution of stars (both single and those affected by binary interactions), in combination with modelling or recipes of binary interactions. The scenarios, or their relative weighting are chosen in such a way as to represent assumed initial parameter distributions (initial primary and secondary mass and orbital period). The outcome of this procedure yields a model for the Galactic population of binaries, that only needs to be normalized in a suitable way to the estimated total number of (binary) stars in the Galaxy.

When studying objects using population synthesis one should keep in mind that several crucial ingredients of the models are poorly known. In particular the effect of binary interaction and mass transfer on the further evolution of the system, the strength of the mass loss (in particular for massive stars), the effect of supernova explosions, and evolution of peculiar stars that can only be formed via binary interactions. In addition, in order to quantitatively compare the models to observed populations, the initial parameter distributions must be known. The distribution of masses for the most massive (primary) component of the binary is usually taken to be the initial mass function (assuming the effect of binaries on the determination of the IMF is dealt with already). For the secondary mass, the observational constraints are of course heavily biased towards similar masses, as otherwise the light of the lower-mass companion (secondary) is buried by the bright primary. Finally the distribution of orbital periods must be patched together from spectroscopic and eclipsing binaries for the shorter periods, via visual binaries to common proper motion pairs for the wider systems. Currently most people use a standard IMF (e.g. Kroupa et al. 1993), a flat mass ratio distribution (i.e. for a given primary mass, each mass lower has equal probability) and a initial semi-major axis distribution that has equal numbers of systems in logarithmic intervals from very close binaries to typically $\sim 10^{4} \mathrm{AU}$. The fact that most people use the same initial conditions is good for comparing results, but the currently used distributions are most likely too simple.

When considering low- and intermediate-mass stars also the normalization is an issue to be considered. Interesting phenomena or types of systems (such as the formation of sdB stars) may occur several Gyr after the formation of the main sequence binary. Therefore the star formation history of the Galaxy becomes important. In most studies the star formation has been taken to be constant or, for old populations, to be a single star burst long ago. In Nelemans et al. (2004) we started to work with a more realistic starformation history, based on the galaxy formation models of Boissier and Prantzos (1999). This is an inside-out star formation model, that peaked early in the history of the Galaxy and has decreased substantially since then. In Fig. 1 we show three snap shots of the formation of the Galaxy, including

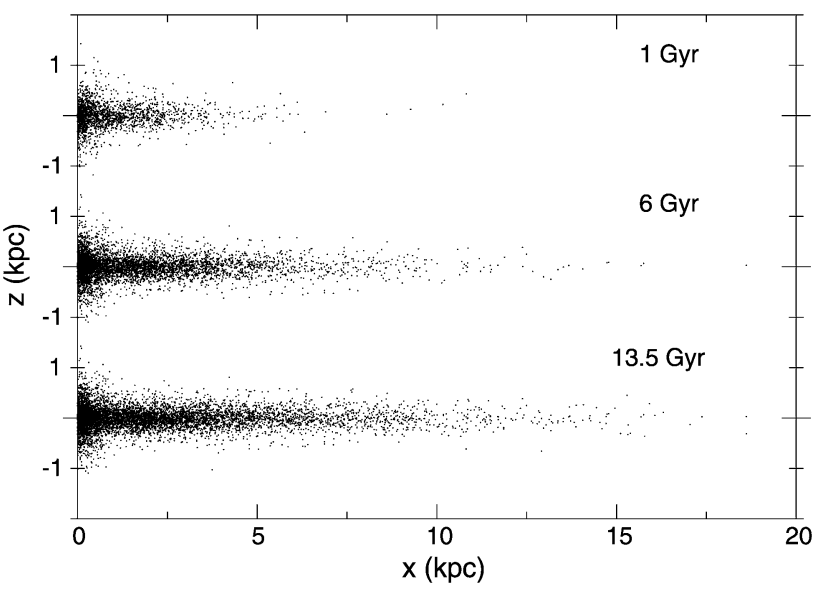

Fig. 1 Representation of our assumed Galactic star-formation model (based on Boissier and Prantzos 1999) for three different Galactic ages. The star formation proceeds from the center, including the Bulge, to the outer disk. The thick disk and Halo are not modelled

Bulge and thin disk. The thick disk and Halo are not included in the model.

\section{Galactic populations of sdB stars}

Han et al. (2002, 2003) and Yungelson and Tutukov (2005) have performed a detailed population synthesis of sdB stars, building on many earlier studies of the formation of helium core burning stars in binaries (e.g. Webbink 1984; Tutukov and Yungelson 1990). So why is it useful to do another population synthesis study? Firstly, because of all the uncertainties in the binary evolution and assumptions about initial parameters and star formation, it is good to compare similar calculations from different groups. In particular, we have concluded from our investigations of the formation of double white dwarfs that a different description for the common-envelope phase was needed in our model (Nelemans et al. 2000), although this has been used by Yungelson and Tutukov (2005) as well. Secondly, as discussed above, we use a rather different star formation history.

The model presented here is basically the same as that discussed in Nelemans et al. (2004), with the exception that for our alternative common envelope we use a value of $\gamma=1.5$, rather than 1.75 . This value fits most of the observed binaries (Nelemans and Tout 2005). Note that we still apply the alternative common envelope only to the cases where none of the two stars is a compact object and the common envelope is triggered by dynamical unstable Rochelobe overflow (rather than a tidal instability). The reason is that, even though in Nelemans and Tout (2005) we conclude that the alternative formalism seems to be able to explain all observed binaries, the motivation for the alternative formalism is the large amount of angular momentum available in binaries with similar mass objects. 
The basic stellar evolution input is described in Portegies Zwart and Verbunt (1996), Nelemans et al. (2001). The stellar evolution tracks are somewhat outdated and we are in the process of updating them. For the moment the evolution of the core of the star is determined by core-mass luminosity relations and following of the growth of the core by shell burning (Nelemans et al. 2001). In our population synthesis calculations, we classify all helium core burning stars that have lost their hydrogen envelope as sdB stars.

The only update for this work is that we allow degenerate helium cores that get exposed via binary interactions and have a core mass close to the mass where the helium flash happens (within $\sim 0.02 \mathrm{M}_{\odot}$, see Han et al. 2002; $\mathrm{Hu}$ et al. 2007) to ignite and become sdB stars (D'Cruz et al. 1996). This feature was not included in our models before, as those cores were assumed to become helium white dwarfs. Because the helium flash for single stars in the stellar models that we use happens at a rather low core mass of 0.446 , this channels produces sdB stars with masses between 0.426 and $0.446 \mathrm{M}_{\odot}$.

\subsection{Results}

I present here the preliminary results of a model for the Galactic population of sdB stars that will be published in more detail elsewhere. We distinguish three classes of objects, sdB stars with main sequence (MS) companions, with white dwarf (WD) companions and single sdB stars.

The number of systems in the Galaxy at present is given in local space densities in Table 1, which is determined by the time and position dependent star-formation rate. We give several different values for different selection effects that may play a role when comparing with observations. First for sdB stars with MS companions, we select those where the $\mathrm{sdB}$ star is at least as bright as the companion in the V-band. Secondly, for both WD and MS companions, we give the density of systems with periods shorter than 10 days, which is about the longest period systems that can be found relatively easily using spectroscopy (e.g. Geier, this volume).

The total space density of $\mathrm{sdB}+$ MS binaries is about a factor of 5 larger than that of sdB + WD binaries, but for the "observable" systems, the space densities are comparable. The space density of double helium white dwarf mergers is roughly the same as the sum of the "observable" binaries. For comparison with other population synthesis studies, I show in the last column of Table 1 the total number of systems in the Galaxy.

In Fig. 2 I show the distribution of periods and companion masses for the sdB + MS binaries (without the selection effects mentioned above, but for a magnitude limited sample). This gives a nice view on the different formation channels (see also Han et al. 2002). The patch at the top, at
Table 1 Local Space densities and total numbers in the Galaxy of different classes of sdB stars and binaries

\begin{tabular}{lll}
\hline Type & $\begin{array}{l}\text { Space density } \\
\left(10^{-7} \mathrm{pc}^{-3}\right)\end{array}$ & $\begin{array}{l}\text { Total number } \\
\left(10^{5}\right)\end{array}$ \\
\hline $\mathrm{sdB}+\mathrm{MS}\left(V_{\mathrm{sdB}} \leq V_{\mathrm{MS}} ; P<10 \mathrm{~d}\right)$ & 1.3 & \\
$\mathrm{sdB}+\mathrm{MS}\left(V_{\mathrm{sdB}} \leq V_{\mathrm{MS}}\right.$ all $\left.\mathrm{P}\right)$ & 7.2 & 16 \\
$\mathrm{sdB}+\mathrm{MS}(\mathrm{all})$ & 23 & \\
$\mathrm{sdB}+\mathrm{WD}(P<10 \mathrm{~d})$ & 3.0 & 6.0 \\
$\mathrm{sdB}+\mathrm{WD}($ all $P)$ & 5.5 & 5.6 \\
He WD mergers & 5.7 & \\
\hline
\end{tabular}

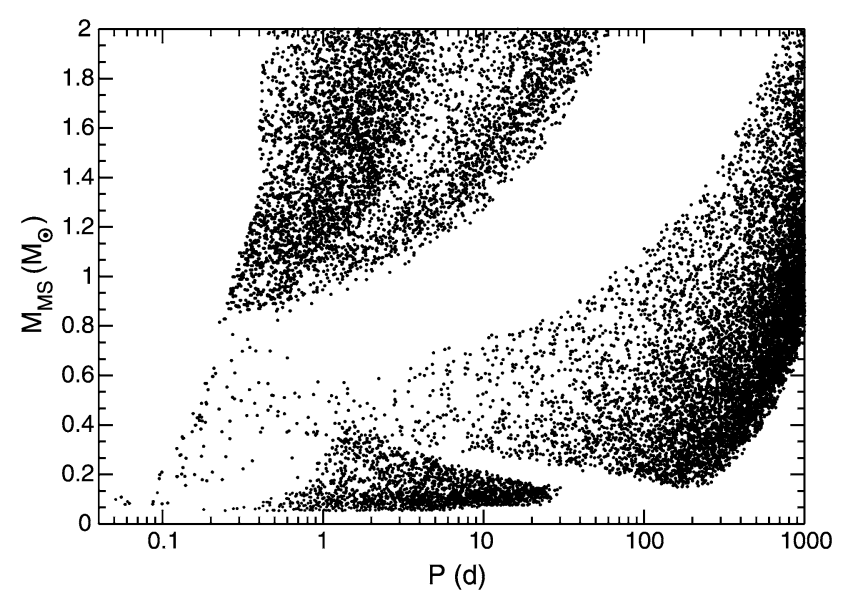

Fig. 2 Period-main sequence mass distribution for the sdB + MS binaries with V-band magnitude of the binary brighter than 20 but no further selection effects. The different formation channels producing this distribution are discussed in the text

relatively short periods and massive companions is a combination of common-envelope ejection of stars with nondegenerate cores, where relatively large separations become a lot shorter (darkest region at shortest periods), and stable Roche-lobe overflow, which in our model can only happen in relatively short period binaries, when the giant fills its Roche lobe in the Hertzsprung gap, widening the orbit (wider periods in that patch). However, all these systems are "unobservable" as the condition $V_{\mathrm{sdB}} \leq V_{\mathrm{MS}}$ effectively limits the mass of the MS companion to below $\sim 0.8 \mathrm{M}_{\odot}$. The patch at the right, at very long periods, is due to our alternative common-envelope prescription for stars near the tip of the red giant branch, leading to mild or hardly any spiral-in. Finally, the triangular patch at the bottom is for stars that enter a common envelope via tidal instability, which we treat with the energy balance in our model (see also Soker, this volume). The low-mass and short period part of that patch could be associated with the HW Vir type binaries of which there are several observed with periods around $0.1 \mathrm{~d}$ (see Heber 2009). The periods on our model are a bit wider (and less concentrated) than those observed, which may indicate that we assume a too large efficiency in the common en- 


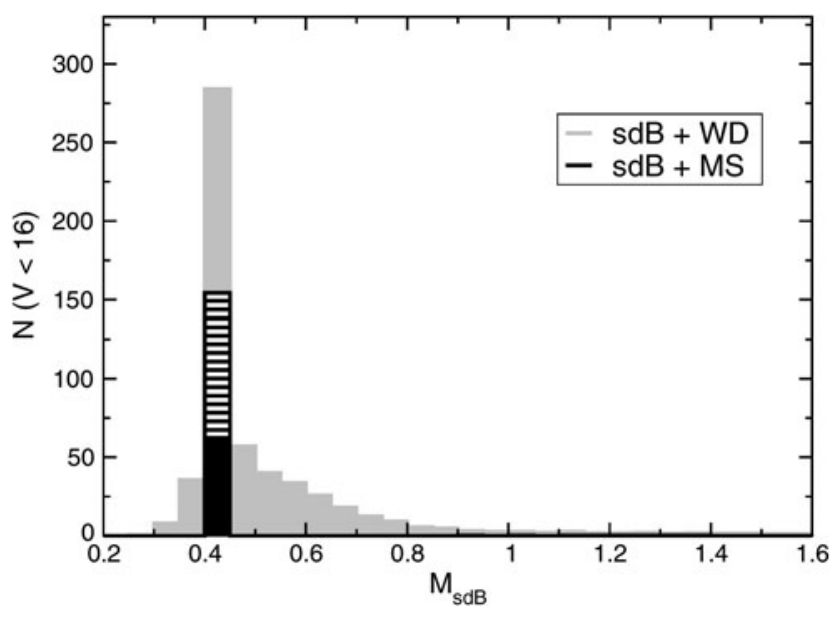

Fig. 3 Mass distribution of sdB stars in close binaries. Dark histogram (total): $\mathrm{sdB}+\mathrm{MS}$ stars (with $V_{\mathrm{sdB}} \leq V_{\mathrm{MS}}$ ). The solid histogram gives the subset of systems with low-mass $\left(\leq 0.15 \mathrm{M}_{\odot}\right)$ companions. Light histogram: sdB + WD systems

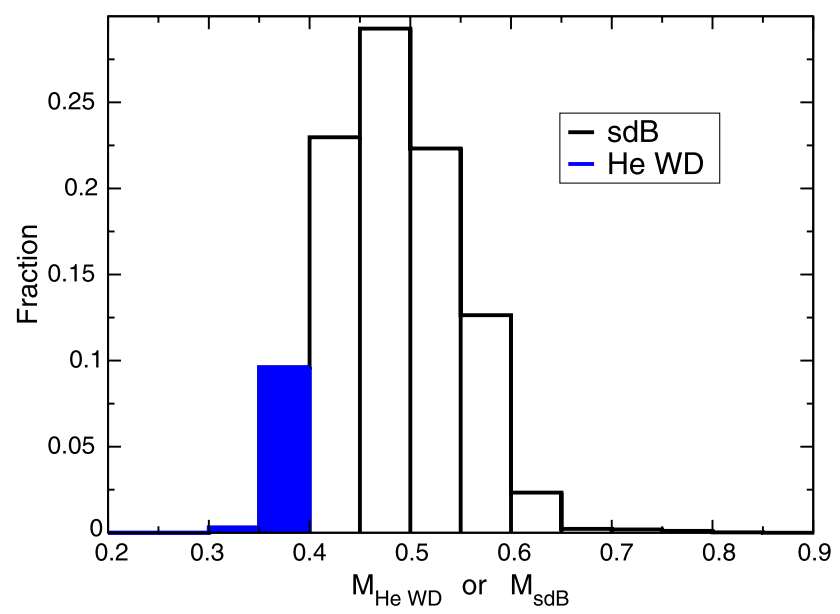

Fig. 4 Mass distribution of the merger products of double helium white dwarfs. The solid histogram below $0.4 \mathrm{M}_{\odot}$ shows those merger products that to not have enough total mass to ignite helium and become an sdB star. Above $0.4 \mathrm{M}_{\odot}$ the ones that do ignite and thus become single sdB stars are shown (open histogram)

velope (we assume $\alpha \lambda=2$ ). A detailed comparison of this model with the observed systems will given in the forthcoming publication.

In Fig. 3 I show the mass distribution of close binary (periods less than 10 days) sdB stars with MS (dark) and WD (light) companions. For the systems with MS companions I only show systems where the estimated V-band magnitude of the sdB stars is smaller or equal to that of the MS star. The dark solid histogram gives the subset of systems with very low-mass $\left(\leq 0.15 \mathrm{M}_{\odot}\right)$ companions, to be compared to the number of HW Vir stars.

The sdB stars with MS companions all come from binary interactions close to the tip of the red giant branch and thus have masses between 0.426 and $0.446 \mathrm{M}_{\odot}$. The ones with WD companions can also originate in more massive stars $\left(M \gtrsim 2 \mathrm{M}_{\odot}\right)$ that ignite helium non-degenerately in their cores and thus can both be lower as well a higher mass than those from the tip of the giant branch. Observable sdB stars with MS companions cannot originate from stars with non-degenerate cores in our models, because these stars are typically more compact and, because of their larger total mass, have more massive envelopes. They could only be unbound by massive MS companions that would outshine the sdB star. In Fig. 4 the distribution of single sdB stars that originate from the merger of two helium white dwarfs (see Sect. 4). The result of mergers are, of course, in general more massive.

\section{Single sdB stars and single helium-core white dwarfs}

Here I further discuss the formation of (apparently) single $\mathrm{sdB} / \mathrm{sdO}$ stars. Different models have been proposed: enhanced mass loss on the red giant branch (D'Cruz et al. 1996), the merger of two helium white dwarfs (e.g. Webbink 1984, for sdO stars) or interactions with low-mass (substellar) companions (Soker 1998). In the remainder we discuss the latter two, as it is unclear if the enhanced wind scenario actually happens.

Interestingly, in both these cases the same mechanism will also produce helium white dwarfs. In the case of the merger when the total mass is too low to ignite the helium (probably around $0.4 \mathrm{M}_{\odot}$, see Han et al. 2002), and in the case of an interaction on the giant branch when the mass of the degenerate core is too low to ignite helium after the hydrogen envelope is lost (as proposed for the observed single helium white dwarfs in Nelemans and Tauris 1998). In both cases the ratio of the formation of single helium white dwarfs and single sdB stars may be different, and these model ratios can then be compared to the observed ratio of single helium white dwarfs to single sdB stars.

In Fig. 4 I show the mass distribution of the double helium white dwarf merger products which shows that in the merger case the vast majority $(\sim 90 \%)$ of objects would ignite helium and thus become $\mathrm{sdB}$ (or sdO) stars. Figure 5, adapted from Nelemans and Tauris (1998), shows the outcome of a common-envelope of a sub-stellar companion with a $1 \mathrm{M}_{\odot}$ red giant for different initial orbital periods and companion masses. If I look at systems in which the sub-stellar companion survives the common envelope and the degenerate helium core is massive enough to ignite and produce an $\mathrm{sdB}$ star (denoted $\mathrm{sdB}+$ planet in the figure), the initial orbital period of the sub-stellar companion is restricted to a very narrow range, say between 1200 and 1300 days. Therefore if we assume as initial period are equally likely, the probability of forming a helium white dwarf is a lot larger than of forming an sdB star. A complicating factor 


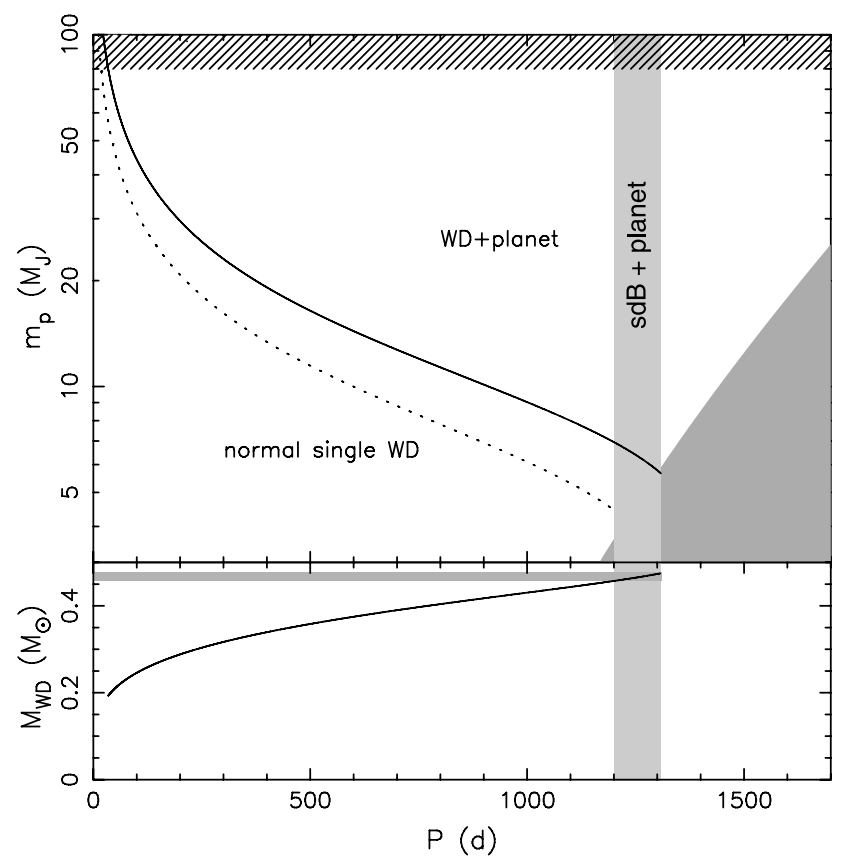

Fig. 5 Outcome of the interaction of a $1 \mathrm{M}_{\odot}$ giant with a sub-stellar companion as function of the initial period and mass of the companion (top). Above the solid line the substellar companion survives, below it evaporates inside the giant envelope. Between the dotted and solid line a significant fraction of the envelope mass is lost and the rest may be lost by stellar winds. The lower panel gives the mass of the core at the moment of the interaction, i.e. the mass of the resulting stars. In order to get an sdB star the core needs to be within a very small mass region (see Sect. 3), so only systems with initial periods between 1200 and 1300 days can produce sdB stars, the rest produces a helium white dwarf. Adapted from Nelemans and Tauris (1998)

is that even if the sub-stellar companion merges with the giant (region denoted normal single WD in the figure), it may cause significant mass loss and spin-up the giant enough that later it will lose its envelope in a stellar wind, exposing the core either when it is still on the red giant branch (yielding a helium white dwarf or an $\mathrm{sdB}$ star depending on the mass of the core), or after it contracted to the horizontal branch following the helium flash (see also Soker 1998; Politano et al. 2008). We therefore included sub-stellar companions in our population synthesis calculation by simply extending the flat mass ratio distribution to include substellar companions, and looked at the ratio of the formation of single helium white dwarfs versus single sdB for interactions on the red giant branch. As the mass ratio distribution at these masses and the fraction of stars with substellar companions are very uncertain, we do not use this calculation to determine the absolute number of systems, but only to assess the ratio of helium white dwarf to $\mathrm{sdB}$ stars formation. As expected, for companions that survive the common envelope, we find a ratio of about 10 for the number of helium white dwarfs versus sdB stars, but even for the mergers that subsequently lose their envelope, we find more helium white

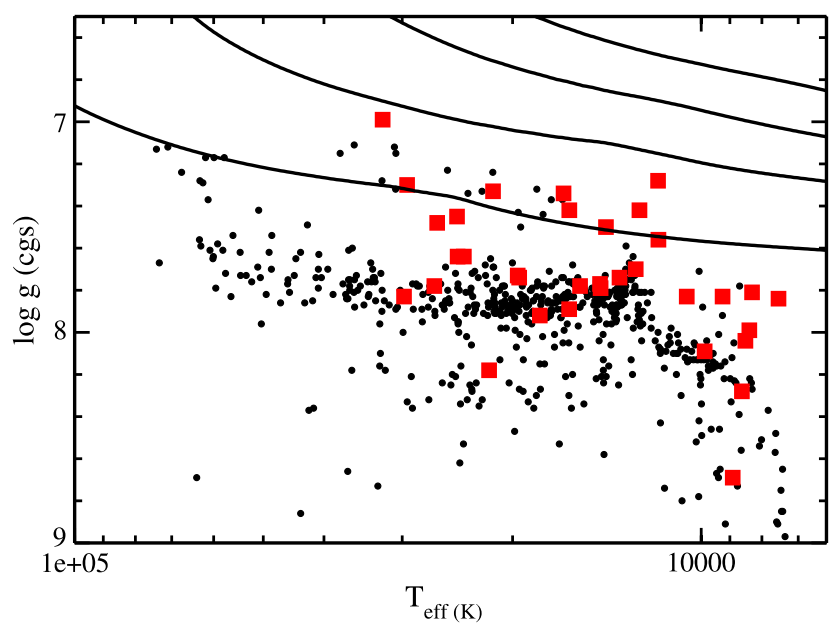

Fig. 6 Effective temperature-surface gravity plot for all white dwarfs observed in the SPY survey (Napiwotzki et al. 2001). The squares show the close binaries, the dots the single white dwarfs. The solid lines give colling tracks for white dwarfs with mass $0.41,0.29,0.24$ and $0.20 \mathrm{M}_{\odot}$ from Althaus et al. (2001)

dwarfs than sdB stars (by about a factor 3). The total ratio comes out at 5.5.

How does this compare with the observational constraints? From the SPY survey (Napiwotzki et al. 2001) we find that 15 out of 636 white dwarfs observed are single helium white dwarfs (see Fig. 6). Assuming a birth rate of white dwarfs (or planetary nebulae) of $3 \times 10^{-12} \mathrm{pc}^{-3} \mathrm{yr}^{-1}$ (Pottasch 1996), this results in a formation rate of single helium white dwarfs of $7.5 \times 10^{-14} \mathrm{pc}^{-3} \mathrm{yr}^{-1}$. For the single $\mathrm{sdB}$ stars we use the estimated birth rate of $4 \times 10^{-14} \mathrm{pc}^{-3} \mathrm{yr}^{-1}$ (Heber 1986), which together with a binary fraction of about $50 \%$ gives a birth rate of $2 \times$ $10^{-14} \mathrm{pc}^{-3} \mathrm{yr}^{-1}$. Because single helium white dwarfs are more common than single sdB stars, they cannot be predominantly formed from mergers. Instead it suggests the single helium white dwarfs are formed from interaction on the red giant branch. If all are formed in that way, the same mechanism will produce single sdB stars at a rate comparable with the observed rate and thus may be responsible for the production of most of the single sdB stars.

However, care should be taken with this reasoning, as there are more ways to produce single sdB stars or single helium white dwarfs which could influence the ratio estimated above. In particular, in Sect. 3.1 I have shown that sdB stars with low-mass companions in wide binaries can easily be more than half of the population (Table 1) even for systems where the $\mathrm{sdB}$ star dominates the (blue) light. Current observational radial velocity studies are not sensitive to these long periods, i.e. would not detect the sdB stars as binaries and thus classify them as single. In addition, single sdB stars may be formed in so-called AM CVn systems (see Nelemans 2005, for a review). Accretion of helium onto a helium white dwarf can lead to ignition at the edge and an 
outside in helium burning front, very likely turning the accreting white dwarf into a helium burning star (but probably without $\mathrm{H}$ atmosphere). A detailed study of this scenario is in preparation. Finally, Justham et al. (2009) have proposed the companions of stars that explode as type Ia supernova to be observable single helium white dwarfs possibly affecting that side of the single helium white dwarf to sdB star ratio. However, the birth rate derived for single helium white dwarfs above, is about a factor ten above the rate of type Ia supernova explosions from single degenerate scenarios, so this scenario is not important for the overall number of single helium white dwarfs.

I conclude that interaction of sub-stellar companions may be a significant contributor to the formation of single sdB stars, but a full detailed study, including all scenarios and proper selection effects is needed to come to firm conclusions.

\section{Massive companions?}

One of the most intriguing results of the last years is the discovery of apparently massive, compact companions to $\mathrm{sdB}$ stars (e.g. Geier et al., this volume and references therein). These masses are derived from the assumption that the $\mathrm{sdB}$ stars in these close binaries should rotate synchronously with the orbit. That means that a combination of the radial velocity, rotational velocity of the $\mathrm{sdB}$ star and an estimate for the sdB star mass, solves the system and thus gives the mass of the companion. The worrying aspect is that all systems found have low inclinations. If the companions are indeed neutron stars or black holes, as is implied by their inferred mass, their fraction is astonishingly large, with several objects in a sample of 31 (Geier et al. this volume). Indeed Yungelson and Tutukov (2005) predict small fractions, and in that study neutron stars and black holes do not receive an asymmetric kick, which typically drastically reduces the surviving close binary population.

Using the same population synthesis model as described before, including asymmetric kicks at the formation of neutron stars (see Portegies Zwart and Yungelson 1999; Nelemans et al. 2001) we find birth rates for sdB stars with neutron stars and black holes of $1.6 \times 10^{-5}$ and $2.2 \times 10^{-7} \mathrm{yr}^{-1}$. This implies about $1 \mathrm{sdB}$-neutron star binary per $100 \mathrm{sdB}$ binaries and $1 \mathrm{sdB}$ - black hole binary per 10,000 sdB binaries. It confirms that finding so many massive companions indeed is not expected, so very exciting if confirmed by higher inclination systems.

\section{Conclusions}

sdB stars are a very useful population for constraining binary evolution theory via population synthesis studies, because they probe very particular evolutionary channels and the number of known objects and their properties is growing rapidly. I have presented preliminary results of a population synthesis study of sdB stars and related objects. Our models show differences with earlier studies, such as (e.g. Yungelson and Tutukov 2005; Han et al. 2003; Tutukov and Yungelson 1990) due to differences in assumptions about binary evolution and the star-formation history of the Galaxy. This means that when proper modelling of the selection effects that govern the detectability of the observed sample is done, we can use the sdB stars to constrain the binary evolution models.

I have shown the promise of comparing the population of sdB stars with their direct cousins, such as helium-core white dwarfs and have compared the birth rates of (apparently) single sdB stars and (apparently) single helium white dwarfs. I conclude that the birth rate of single helium white dwarfs is substantially larger than that of single sdB stars, suggesting that at least for the helium white dwarfs interactions on the red giant branch with sub-stellar companions are important.

Finally we have calculated the birth rate of sdB stars with neutron star and black hole companions and concluded that the models predict substantially smaller fractions ( 1 and 0.01 per cent respectively) than found in short period $\mathrm{sdB}$ binaries, assuming the sdB star is in co-rotation with the orbit. This either means that assumption is not correct, or much more interestingly, that there are many mode sdB stars with massive compact companions in close binaries than the models predict.

Acknowledgements It is a pleasure to thank Lev Yungelson, Simon Portegies Zwart, Tom Marsh and Ralf Napiwotzki for much enjoyed collaborations that have contributed to the material presented here.

Open Access This article is distributed under the terms of the Creative Commons Attribution Noncommercial License which permits any noncommercial use, distribution, and reproduction in any medium, provided the original author(s) and source are credited.

\section{References}

Althaus, L.G., Serenelli, A.M., Benvenuto, O.G.: Mon. Not. R. Astron. Soc. 323, 471 (2001)

Belczynski, K., Kalogera, V., Rasio, F.A., Taam, R.E., Zezas, A., Bulik, T., Maccarone, T.J., Ivanova, N.: Astrophys. J. Suppl. 174, 223 (2008)

Boissier, S., Prantzos, N.: Mon. Not. R. Astron. Soc. 307, 857 (1999)

D’Cruz, N.L., Dorman, B., Rood, R.T., O'Connell, R.W.: Astrophys. J. 466, 359 (1996)

de Kool, M.: Astrophys. J. 358, 189 (1990)

Dewey, R.J., Cordes, J.M.: Astrophys. J. 321, 780 (1987)

Fontaine, G., Brassard, P., Charpinet, S., Green, E.M., Chayer, P., Randall, S.K., van Grootel, V.: In: Heber, U., Jeffery, C.S., Napiwotzki, R. (eds.) Hot Subdwarf Stars and Related Objects. Astronomical Society of the Pacific Conference Series, vol. 392, p. 231 (2008)

Fryer, C., Burrows, A., Benz, W.: Astrophys. J. 496, 333 (1998) 
Han, Z., Podsiadlowski, P., Maxted, P.F.L., Marsh, T.R., Ivanova, N.: Mon. Not. R. Astron. Soc. 336, 449 (2002)

Han, Z., Podsiadlowski, P., Maxted, P.F.L., Marsh, T.R.: Mon. Not. R. Astron. Soc. 341, 669 (2003)

Han, Z., Podsiadlowski, P., Lynas-Gray, A.E.: Mon. Not. R. Astron. Soc. 380, 1098 (2007)

Heber, U.: Astron. Astrophys. 155, 33 (1986)

Heber, U.: Annu. Rev. Astron. Astrophys. 47, 211 (2009)

Hu, H., Nelemans, G., Østensen, R., Aerts, C., Vučković, M., Groot, P.J.: Astron. Astrophys. 473, 569 (2007)

Hurley, J.R., Tout, C.A., Pols, O.R.: Mon. Not. R. Astron. Soc. 329, 897 (2002)

Justham, S., Wolf, C., Podsiadlowski, P., Han, Z.: Astron. Astrophys. 493, 1081 (2009)

Kolb, U.: Astron. Astrophys. 271, 149 (1993)

Kornilov, V.G., Lipunov, V.M.: Soviet Astron. 27, 334 (1983)

Kroupa, P., Tout, C.A., Gilmore, G.: Mon. Not. R. Astron. Soc. 262, 545 (1993)

Lipunov, V.M., Postnov, K.A.: Astrophys. Space Sci. 145, 1 (1988)

Napiwotzki, R., Christlieb, N., Drechsel, H., Hagen, H.J., Heber, U., Homeier, D., Karl, C., Koester, D., Leibundgut, B., Marsh, T.R., Moehler, S., Nelemans, G., Pauli, E.M., Reimers, D., Renzini, A., Yungelson, L.: Astron. Nachr. 322, 411 (2001)

Nelemans, G.: In: ASP Conf. Ser. 330: The Astrophysics of Cataclysmic Variables and Related Objects, p. 27 (2005). astro-ph/0409676

Nelemans, G., Tauris, T.M.: Astron. Astrophys. 335, 85 (1998)
Nelemans, G., Tout, C.A.: Mon. Not. R. Astron. Soc. 356, 753 (2005)

Nelemans, G., Yungelson, L.R., Portegies Zwart, S.F.: Astron. Astrophys. 375, 890 (2001)

Nelemans, G., Verbunt, F., Yungelson, L.R., Portegies Zwart, S.F.: Astron. Astrophys. 360, 1011 (2000)

Nelemans, G., Yungelson, L.R., Portegies Zwart, S.F., Verbunt, F.: Astron. Astrophys. 365, 491 (2001)

Nelemans, G., Yungelson, L.R., Portegies Zwart, S.F.: Mon. Not. R. Astron. Soc. 349, 181 (2004)

Politano, M., Taam, R.E., van der Sluys, M., Willems, B.: Astrophys. J. 687, 99 (2008)

Pols, O.R., Marinus, M.: Astron. Astrophys. 288, 475 (1994)

Portegies Zwart, S.F., Verbunt, F.: Astron. Astrophys. 309, 179 (1996)

Portegies Zwart, S.F., Yungelson, L.R.: Mon. Not. R. Astron. Soc. 309, 26 (1999)

Pottasch, S.R.: Astron. Astrophys. 307, 561 (1996)

Soker, N.: Astrophys. J. 116, 1308 (1998)

Tutukov, A.V., Yungelson, L.R.: Soviet Astron. 34, 57 (1990)

Vanbeveren, D., van Bever, J., de Donder, E.: Astron. Astrophys. 317, 487 (1997)

Webbink, R.F.: Astrophys. J. 277, 355 (1984)

Yungelson, L.R., Tutukov, A.V.: In: van der Hucht, K.A., Hidayat, B. (eds.) Wolf-Rayet Stars and Interrelations with Other Massive Stars in Galaxies. IAU Symposium, vol. 143, p. 459 (1991)

Yungelson, L.R., Tutukov, A.V.: Astron. Rep. 49, 871 (2005) 